Journal of Nonlinear Mathematical Physics

Vol. 4 (1997) No. 1/2 (p. 89-97)

http://staff.www.ltu.se/ norbert/home_journal/electronic/v4n1-2.html

\title{
Symmetries of vector exterior differential systems and the inverse problem in second-order Ostrohrads'kyj mechanics
}

\author{
R. Ya. Matsyuk \\ email: romko.b.m@gmail.com \\ http://www.iapmm.Iviv.ua/12/eng/files/st_files/matsyuk.htm \\ Institute for Applied Problems in Mechanics and Mathematics
}

\begin{abstract}
Symmetries of variational problems are considered as symmetries of vector bundle valued exterior differential systems. This approach is then applied to third order ordinary variational equations of motion of the semi-classical spinning particle.
\end{abstract}

It is common in the similarity theories of differential equations to consider transformations which leave invariant some prolonged manifolds in jet-spaces of appropriate order within which the integral manifolds of a given system of partial differential equations lie [1]. From this invariance it follows that the solutions of the system in charge transform but into some other solutions. However, we are not sure enough that the prolonged manifold is "densely" covered by the integral manifolds. If not, then some additional symmetries may occur. In this paper we consider a symmetry of a differential equation be defined in general as a generator of such a transformation, which carries every solution into nothing more than some other solution.

Then we reformulate this into slightly different language of the algebraic invariance of some vector valued exterior differential system. This has an advantage that we can use Lagrange multiplies method to solve the problem. In the case of ordinary differential equations the two approaches (algebraic and the general one) coincide due to the completeness of Pfaff systems (that is that every differential form, annulled by every solution of the system expands by the Lagrange multiplies into the elements of the differential ideal, generated by the system itself).

The Euler-Lagrange equations of the variational calculus (of arbitrary order) naturally fall into the framework of vector bundle valued exterior differential systems when the dependent variables are globally segregated from the independent ones [2]. 
This is the case of the field theory. In general such segregation depends on the local chart $\mu: M \rightarrow \mathbb{R}^{p} \times \mathbb{R}^{q}$ which constitutes a way of introducing a fibred structure in the manifold $M$. In applying infinitesimal considerations we can use this structure every time the manifold $M$ is not endowed by a fibred structure in an intrinsic manner. If $Y$ denotes a fibred manifold over a base $Z$, we take $Y_{s} \stackrel{\text { def }}{=} J_{s} Y$ to mean the space of all the jets of order $s$ of cross-sections of the surmersion $\pi: Y \rightarrow Z$. Then $\mu_{J}: Y_{s} \rightarrow J_{s}\left(\mathbb{R}^{p} \times \mathbb{R}^{q}\right) \approx J_{s}\left(\mathbb{R}^{p}, \mathbb{R}^{q}\right)$ will denote the standard $s$-order prolongation of the local chart $\mu$.

1. Vector bundle valued differential systems. Let $E \rightarrow B, E_{1} \rightarrow B_{1}, F \rightarrow$ $X, F_{1} \rightarrow X_{1}$, and $E^{\prime} \rightarrow B$ be some fibre bundles, let $\Gamma(E)$ denote the set of smooth cross-sections of the fibred manifold $E$ and let $\mathfrak{F}(B)$ stand for the ring of smooth functions defined on the manifold $B$. We shall denote the tensor product of the inverse image of vector bundles with respect to some manifold morphisms $f$ : $B \rightarrow X$ and $f_{1}: B \rightarrow X_{1}$ by $\otimes_{B}$. If the manifold $B$ is fibred over some manifold $N$, the semi-basic differential forms over the manifold $B$ with values in the vector bundle $F$ are defined as smooth cross-sections of the vector bundle $\wedge T^{*} N \otimes_{B} \mathfrak{F}$. They constitute an $\mathfrak{F}(B)$-submodule, denoted by $\mathfrak{F}_{B}(N, F)$, of the graded module $\mathfrak{F}(B, F)=\Gamma\left(\wedge T^{*} B \otimes_{B} F\right)$. Every bundle homomorphism $h: F \rightarrow F_{1}$ over the base homomorphism $\underline{h}: X \rightarrow X_{1}$ satisfying $f_{1}=\underline{h} \circ f$ obviously defines the moduli homomorphism $h_{\#}: \mathfrak{F}(B, F) \rightarrow \mathfrak{F}\left(B, F_{1}\right)$. The canonical vector bundle pairing $E \times \operatorname{Hom}\left(E, E^{\prime}\right) \rightarrow E^{\prime} \quad$ defines an exterior product $\wedge: \mathfrak{F}(B, E) \times \mathfrak{F}\left(B, E^{*} \otimes E^{\prime}\right) \rightarrow$ $\mathfrak{F}\left(B, E^{\prime}\right)$. If we think of a cross-section $\varphi \in \mathfrak{F}^{0}\left(B, E^{*} \times E^{\prime}\right)$ as a $B$-morphism $\widehat{\phi}: E \rightarrow E^{\prime}$, then $\widehat{\phi}_{\#} \boldsymbol{\omega}=\boldsymbol{\omega} \wedge \boldsymbol{\varphi}$ for any $\boldsymbol{\omega} \in \mathfrak{F}(B, E)$.

The vector bundle $\operatorname{End}(E)$ is a bundle of algebras with the composition rule that of the superposition of endomorphism. This fibrewise composition defines the structure of a graded non-commutative algebra in the $\mathfrak{F}(B)$ module of $\operatorname{End}(E)$ valued differential forms which we denote by $\mathfrak{F}(E$, End $E)$. The vector bundle $E$ may be viewed as the bundle of $\operatorname{End}(E)$-moduli with respect to the pairing $E \times$ End $(E) \rightarrow E$. This pairing makes the $\mathfrak{F}(B)$-module $\mathfrak{F}(B, E)$ into a graded module over the algebra $\mathfrak{F}(B$, End $E)$.

Now let $\mathrm{h}: E_{1} \rightarrow E$ be a vector bundle homomorphism over a base morphism $g: B_{1} \rightarrow B$ and let $\mathrm{h}_{1}$ denote the corresponding induced $B_{1}$-homomorphism of vector bundles $\mathrm{h}_{1}: E_{1} \rightarrow g^{-1} E$. The dual vector bundle homomorphism $\mathrm{h}_{1}{ }^{*}$ : $\left(g^{-1} E\right)^{*} \rightarrow E_{1}{ }^{*}$ denotes, by virtue of identification $\left(g^{-1} E\right)^{*} \approx g^{-1}\left(E^{*}\right)$, some $g$-comorphism $\mathrm{h}^{*}: g^{-1}\left(E^{*}\right) \rightarrow E_{1}{ }^{*}$. If set $E=T B, E_{1}=T B_{1}$, one gets the action $\mathrm{h}^{\#}$ of $\mathrm{h}$ on the ordinary differential forms on $B$. If $\alpha_{g}$ stand for the inverse image of the cross-section $\alpha \in \mathfrak{F}(B, \mathbb{R})$, then $\mathrm{h}^{\#} \alpha=(\wedge \mathrm{h})^{*} \circ \alpha_{g} \in \mathfrak{F}\left(B_{1}, \mathbb{R}\right)$. Given some cross-section $\boldsymbol{\omega} \in \mathfrak{F}(B, E)$ and recalling the notion of its inverse image $\boldsymbol{\omega}_{g} \in \Gamma\left(\wedge T^{*} B \otimes_{B_{1}} E\right)$, the differential form $g^{\star} \boldsymbol{\omega} \in \mathfrak{F}\left(B_{1}, E\right)$ is being defined by the superposition of mappings: $g^{\star} \boldsymbol{\omega}=\left((\wedge T g)^{*} \otimes\right.$ id $) \circ \boldsymbol{\omega}_{g}$. If the morphism $g$ has a canonical prolongation to a vector bundle $g$-comorphism $\mathrm{k}: g^{-1} E \rightarrow E_{1}$, then the notation $g^{\#} \boldsymbol{\omega}$ will mean the differential form $\mathbf{k}_{\#} g^{\star} \boldsymbol{\omega}=\left((\wedge T g)^{*} \otimes \mathbf{k}\right) \circ \boldsymbol{\omega}$.

The functor $g^{\star}$ is consistent with the tensor product and contraction operations. 
Let throughout this paragraph the manifold $B$ be parallelizable and the module $\Gamma(E)$ be free. Then, if $\boldsymbol{\omega}=\alpha \otimes \boldsymbol{\gamma} \in \mathfrak{F}(B, E) \approx \mathfrak{F}(B, \mathbb{R}) \otimes \Gamma(E)$ and if $\boldsymbol{\Omega}=\beta \otimes \boldsymbol{\varphi} \in$ $\mathfrak{F}\left(B, E^{*} \otimes E^{\prime}\right) \approx \mathfrak{F}(B, \mathbb{R}) \otimes \Gamma\left(E^{*} \otimes E^{\prime}\right)$, then for the inverse images of the cross-sections $\boldsymbol{\omega}, \boldsymbol{\Omega}$, and $\boldsymbol{\omega} \wedge \boldsymbol{\Omega}=(\alpha \wedge \beta) \otimes\left\langle\gamma_{g}, \boldsymbol{\varphi}\right\rangle$, it is true that $(\boldsymbol{\omega} \wedge \boldsymbol{\Omega})_{g}=\left(\alpha_{g} \wedge \beta_{g}\right) \otimes\left\langle\gamma_{g}, \boldsymbol{\varphi}_{g}\right\rangle \in$ $\Gamma\left(\wedge T^{*} B \otimes_{B_{1}} E^{\prime}\right)$. Composing with $(\wedge T g)^{*}$ one gets $g^{\star}(\boldsymbol{\omega} \wedge \boldsymbol{\Omega})=g^{\star} \boldsymbol{\omega} \wedge g^{\star} \boldsymbol{\Omega}$.

A one-form $\vartheta \in \mathfrak{F}^{1}(B, T B)$ may be thought of as a $B$-homomorphism of vector bundles $\hat{\theta}: T B \rightarrow T B$; then the dual homomorphism $\hat{\theta}^{*}: T^{*} B \rightarrow T B$ acts over the cross-sections from $\Gamma\left(T^{*} B\right) \equiv \mathfrak{F}^{1}(B, \mathbb{R})$ in an obvious manner and is being extended to $\mathfrak{F}(B, \mathbb{R})$ as a differentiation of degree 0 acting trivially on the ring of functions. We denote this extended action by $\boldsymbol{\vartheta} \bar{\wedge}$. Again within local considerations for a parallelizable $B$ and $\Gamma(E)$ being free, we can identify $\mathfrak{F}(B$, End $E)$ with the tensor product $\mathfrak{F}(B, \mathbb{R}) \otimes_{\mathfrak{F}(B)} \Gamma($ End $E)$ and define $\boldsymbol{\vartheta} \bar{\wedge}(\alpha \otimes \boldsymbol{\varphi})=(\boldsymbol{\vartheta} \bar{\wedge} \alpha) \otimes$ id $(\boldsymbol{\varphi})$. Consider the ideal $\mathfrak{I} \equiv \mathfrak{I}(B) \equiv \mathfrak{I}(\boldsymbol{\vartheta})=\sum_{d>0} \mathfrak{I}^{(d)}=\boldsymbol{\vartheta} \bar{\wedge} \mathfrak{F}(B, \mathbb{R})$ in the algebra $\mathfrak{F}(B, \mathbb{R})$. Consider also the $\mathfrak{F}(B, \mathbb{R})$-submodule $\mathfrak{I}(B$, End $E)=\boldsymbol{\vartheta} \bar{\wedge} \mathfrak{F}(B$, End $E)$ in the $\mathfrak{F}(B, \mathbb{R})$-module $\mathfrak{F}(B$, End $E)$. We denote by $\mathfrak{I}(B, E)$ the submodule $\mathfrak{F}(B, E) \wedge$ $\mathfrak{I}$ in the module $\mathfrak{F}(B, E)$ over the algebra $\mathfrak{F}(B, \mathbb{R})$. It is clear that $\mathfrak{I}(B, E)$ is also a submodule over the algebra $\mathfrak{F}(B$, End $E)$ and that $\mathfrak{I}(B, E)=\mathfrak{F}(B, E) \wedge$ $\mathfrak{I}(B$, End $E)$. In fact, $\mathfrak{I}(B, E)$ is generated over $\mathfrak{F}(B, \mathbb{R})$ by the $\mathfrak{F}^{0}(B$, End $E)$ submodule of one-forms

$$
\mathfrak{P}(B, E)=\boldsymbol{\vartheta} \bar{\wedge} \mathfrak{F}^{1}(B, E)=\mathfrak{F}^{0}(B, E) \wedge \mathfrak{P},
$$

where $\mathfrak{P} \equiv \mathfrak{P}(B) \equiv \mathfrak{I}^{(1)}=\boldsymbol{\vartheta} \wedge \mathfrak{F}^{1}(B, \mathbb{R})$ is an $\mathfrak{F}(B)$-submodule, that is the ordinary Pfaff system.

Definition 1 An exterior differential system $\mathfrak{S} \equiv \mathfrak{S}(F)$ (with values in the vector bundle $F)$ is an $\mathfrak{F}(B$, End $F)$-submodule of the module $\mathfrak{F}(B, F)$. A Pfaff system is an exterior differential system, generated by one-forms. Let a manifold $Z$ be given. We call the germ of an immersion $\sigma: Z \rightarrow B$ be a solution of the exterior differential system $\mathfrak{S}$ if $\sigma^{\star} \mathfrak{S}=0$. Two exterior differential systems (in general, over different manifolds) are called equivalent if the sets of their solutions coincide (in general, are isomorphic).

Let over a manifold $B$ be given an exterior differential system $\mathfrak{S}$ with values in the vector bundle $E$ and an exterior differential system $\mathfrak{S}^{\prime}$ with values in the vector bundle $E^{\prime}$ and let $\operatorname{sol}(\mathfrak{S})$ denote the set of solutions of the system $\mathfrak{S}$. If $\mathfrak{S} \wedge \mathfrak{F}\left(B, E^{*} \otimes E^{\prime}\right) \supset \mathfrak{S}^{\prime}$, then $\operatorname{sol}(\mathfrak{S}) \subset \operatorname{sol}\left(\mathfrak{S}^{\prime}\right)$.

Definition 2 Exterior differential systems $\mathfrak{S}$ and $\mathfrak{S}^{\prime}$ are algebraically equivalent, if at the same time both $\mathfrak{S} \wedge \mathfrak{F}\left(B, E^{*} \otimes E^{\prime}\right) \supset \mathfrak{S}^{\prime}$ and $\mathfrak{S}^{\prime} \wedge \mathfrak{F}\left(B, E^{*} \otimes E\right) \supset \mathfrak{S}$.

Lemma 1 Two Pfaff systems which are algebraically equivalent, are equivalent.

This follows from the completeness of Pfaff systems.

Let $\widetilde{\mathfrak{S}}$ denote the image of the exterior differential system $\mathfrak{S}$ under the projection $j: \mathfrak{F}(B, E) \rightarrow \mathfrak{F}(B, E) / \mathfrak{I}(B, E)$. In view of the inclusion $\mathfrak{I}(B, E) \wedge \mathfrak{F}\left(B, E^{*} \otimes E^{\prime}\right) \subset$ 
$\mathfrak{I}\left(B, E^{\prime}\right)$ the action $\wedge$ of the elements from $\mathfrak{F}\left(B, E^{*} \otimes E^{\prime}\right)$ over the quotient module is defined and if for this action $\mathfrak{S} \wedge \mathfrak{F}\left(B, E^{*} \otimes E^{\prime}\right) \supset \mathfrak{S}^{\prime}$, then also $\widetilde{\mathfrak{S}} \wedge \mathfrak{F}\left(B, E^{*} \otimes E^{\prime}\right) \supset$ $\widetilde{\mathfrak{S}^{\prime}}$.

Definition 3 A solution of the system $(\mathfrak{S}, \boldsymbol{\vartheta})$ is a germ of an immersion $\sigma: Z \rightarrow$ $B$, such that $\sigma^{\star} j^{-1}(\widetilde{\mathfrak{S}})=0$.

Let over the manifold $B_{1}$ be specified a differential form $\boldsymbol{\vartheta}_{1} \in \mathfrak{F}\left(B_{1}, T B_{1}\right)$ such that $g^{\star} \boldsymbol{\vartheta}=\boldsymbol{\vartheta}_{1} \bar{\Lambda} \boldsymbol{\varrho} \in \mathfrak{P}\left(B_{1}, g^{-1} T B\right)$ for some $\varrho \in \mathfrak{F}\left(B_{1}, T B\right)$. Taking into account the definitions of the actions $g^{\star}, \boldsymbol{\vartheta}_{1} \bar{\wedge}$ and identifying the differential forms $\boldsymbol{\vartheta}, \boldsymbol{\vartheta}_{1}$, and $\varrho$ with the corresponding vector bundle homomorphisms $\widehat{\theta}, \widehat{\theta}_{1}$ and $\widehat{\rho}: T B_{1} \rightarrow g^{*} T B$ we have $\widehat{\theta}_{g} \circ(T g)_{1}=\widehat{\rho} \circ \widehat{\theta}_{1}$, where $\widehat{\theta}_{g}$ is the inverse image of the homomorphism $\widehat{\theta}$ with respect to the morphism $g$. For the conjugated homomorphisms we also have $(T g)^{*} \circ \widehat{\theta}_{g}^{*}=\widehat{\theta}_{1}^{*} \circ \widehat{\rho}^{*}$.

In view of the definition of the action $\widehat{\rho}^{\#}$ on the differential form $\alpha \in \mathfrak{F}^{1}(B, \mathbb{R})$ it holds that

$$
g^{\star}(\boldsymbol{\vartheta} \bar{\wedge} \alpha)=(T g)^{*} \circ \widehat{\theta}_{g}{ }^{*} \circ \alpha_{g}=\widehat{\theta}_{1}{ }^{*} \circ \widehat{\rho}^{*} \circ \alpha_{g}=\boldsymbol{\vartheta}_{1} \bar{\wedge} \widehat{\rho}^{*} \alpha \in \mathfrak{P}_{1} \equiv \mathfrak{P}\left(B_{1}\right) .
$$

It follows that $g^{\star} \mathfrak{P} \subset \mathfrak{P}_{1}$, thus $g^{\star} \mathfrak{I} \subset \mathfrak{I}_{1}$ and also

$$
g^{\star} \mathfrak{I}(B, E)=g^{\star} \mathfrak{F}(B, E) \wedge g^{\star} \mathfrak{I} \subset \mathfrak{F}\left(B_{1}, g^{-1} E\right) \wedge \mathfrak{I}\left(B_{1}, g^{-1} E\right),
$$

so the action $g^{\star}$ of the homomorphism $g$ over the quotient module $\mathfrak{F}(B, E / \mathfrak{I}(B, E))$ is defined.

2. The Lie derivative. Let $w: B \rightarrow W$ be a morphism of manifolds and let $w_{t}$ denote its deformation. Then $\dot{w}_{t}(0): B \rightarrow T W$ is a lift of the morphism $w$. If the manifold $W$ is fibred over the manifold $B$ and if every $w_{t}$ is a cross-section, then $\dot{w}_{t}(0)$ belongs to the vertical tangent bundle $V W \subset T W$. Let $\xi$ and $\bar{\eta}$ be some vector fields on the manifolds $B$ and $W$ respectively. The Lie derivative [3] of the morphism $w$ with respect to the pair of vector fields $\xi, \bar{\eta}$ is defined by the expression $\mathbf{L}_{\xi, \bar{\eta}} w=(T w) \circ \xi-\bar{\eta} \circ w$. If $w$ is a cross-section and if the vector field $\bar{\eta}$ is projectible onto the vector field $\xi$, then the Lie derivative $\mathbf{L}_{\xi, \bar{\eta}} w$ belongs to $V W$. Let under this assertions $\exp _{B} t \bar{\eta}$ denote the one-parameter induced family of diffeomorphism over $B$, corresponding to $\exp t \bar{\eta}$ and let

$$
w_{t}=\exp _{B}(-t \bar{\eta}) \circ w_{\exp t \xi}=\exp (-t \bar{\eta}) \circ w \circ \exp t \xi .
$$

Differentiating at $t=0$ we obtain

$$
\dot{w}_{t}(0)=-(\exp t \bar{\eta}) \circ w+(T w) \circ(\exp t \xi)^{\cdot}=\mathbf{L}_{\xi, \bar{\eta}} w .
$$

If the fibre bundle $W$ is a vector bundle, then $V W \approx W \times_{B} W$, the first component of $\dot{w}_{t}(0)$ coincides with the initial cross-section $w$, and the second one is identified with the Lie derivative $\mathbf{L}_{\xi, \bar{\eta}} w$ itself. In a special case setting $W=\wedge T^{*} B \otimes E$ and taking $\bar{\eta}$ to be build up from the standard lift to the cotangent bundle of the vector field $\xi$ together with some vector field $\eta$ on $E$, projectible into $\xi$, we agree with truncated notation of $\mathbf{L}_{\xi, \eta} \stackrel{\text { def }}{=} \mathbf{L}_{\xi, \bar{\eta}}$. 
Definition $4 A$ diffeomorphism $g: B \rightarrow B$ is called a symmetry (vs an algebraic symmetry) of the exterior differential system $\mathfrak{S}(E)$, if the exterior differential systems $g^{\star} \mathfrak{S}$ and $\mathfrak{S}$ itself are equivalent (vs algebraically equivalent). A vector field $\xi$ on the manifold $B$ is called an infinitesimal symmetry (vs an algebraic infinitesimal symmetry) of the exterior differential system $\mathfrak{S}$ if for all t the transformation $\exp t \xi$ is a symmetry (vs an algebraic symmetry) of the system $\mathfrak{S}$.

That $\xi$ is an algebraic infinitesimal symmetry of the exterior differential system $\mathfrak{S}$ means that

$$
(\exp t \xi)^{\star} \mathfrak{S} \subset \mathfrak{S} \wedge \mathfrak{F}\left(B, E^{*} \otimes(\exp t \xi)^{-1} E\right) .
$$

In particular, whatever the vector field $\eta \in \Gamma(T E)$, projectible into the vector field $\xi$ be, the inclusions

$$
\begin{aligned}
& (\exp -t \eta)_{B}(\exp t \xi)^{-1} \mathfrak{S} \subset \mathfrak{S} \wedge \mathfrak{F}\left(B, E^{*} \otimes(\exp t \xi)^{-1} E \wedge \mathfrak{F}^{0}\left(B,(\exp t \xi)^{-1 \star}\left(E^{*} \otimes E\right)\right)\right. \\
& \subset \mathfrak{S} \wedge \mathfrak{F}\left(B, E^{*} \otimes E\right)=\mathfrak{S}
\end{aligned}
$$

hold. By differentiating with respect to the parameter $t$ we conclude that if $\xi$ is the infinitesimal symmetry of the system $\mathfrak{S}$, then

$$
\mathbf{L}_{\xi, \eta} \mathfrak{S}=\frac{d}{d t}(\exp t \xi)^{\star} \mathfrak{S}(0) \subset \mathfrak{S}
$$

for every vector field $\eta$, projectible into the field $\xi$. In the case of a trivial vector bundle $E$, one can put $\eta=(\xi, 0)$ and use the notation $\mathbf{L}_{\xi, 0}$ in place of $\mathbf{L}_{\xi,(\xi, 0)}$.

Definition $5 A$ diffeomorphism $g: B \rightarrow B$ is called a symmetry (vs algebraic symmetry) of the system $(\mathfrak{S}, \boldsymbol{\vartheta})$, if $g^{\star} \mathfrak{I} \subset \mathfrak{I}$ and if the exterior differential systems $g^{\star} \widetilde{\mathfrak{S}}$ and $\widetilde{\mathfrak{S}}$ itself are equivalent (vs algebraically equivalent).

An (algebraic symmetry of system $(\mathfrak{S}, \boldsymbol{\vartheta})$ is symmetry (vs an algebraic symmetry) of the exterior differential system $\mathfrak{S}+\mathfrak{I}(B, E)$ and vice versa. In that case $\mathbf{L}_{\xi} \mathfrak{I} \subset \mathfrak{I}$ and $\mathbf{L}_{\xi, \eta} \widetilde{\mathfrak{S}} \subset \widetilde{\mathfrak{S}}$, that is

$$
\mathbf{L}_{\xi, \eta} \mathfrak{S} \subset \mathfrak{S}+\mathfrak{I}(B, E) .
$$

3. Symmetries of the Euler-Lagrange equations. On the manifold $Y_{s+1}$ of the jets of order $s+1$ of the cross-sections of the fibred manifold $\pi: Y \rightarrow Z$ there exists a canonical contact differential form $\boldsymbol{\vartheta}_{s} \in \mathfrak{F}_{s+1}\left(Y_{s}, V_{s}\right)$, semi-basic with respect to the projection $\pi_{s}^{s+1}: Y_{s+1} \rightarrow Y_{s}$ which takes values in the vector bundle $V_{s}$ of vertical tangent vectors to the surmersion $\pi^{s}: Y_{s} \rightarrow Z$ and such that Pfaff system $\mathfrak{P}_{s}=\boldsymbol{\vartheta}_{s} \wedge \mathfrak{F}_{s+1}^{1}\left(Y_{s}, \mathbb{R}\right)$ is nothing but the Cartan co-distribution. (We use subscript $s$ instead of $Y_{s}$ where possible). For an open set $U_{s} \subset Y_{s}$ and applying the projection $\pi_{v}^{s}: Y_{s} \rightarrow Y_{v}$ let us put $\mathfrak{I}_{v}\left(U_{s}\right)=\mathfrak{I}\left(\left.\pi_{v}^{s}\right|_{U_{s}} \boldsymbol{\vartheta}_{v}\right)$ and let $v_{J}: Y_{s} \rightarrow J\left(\mathbb{R}^{p}, \mathbb{R}^{q}\right)$ be the canonical lift of a local fibred chart $v$ on the manifold $Y$. A lagrangian is an element $\widetilde{\lambda}$ of the quotient sheaf, generated by the quotient moduli $\mathfrak{F}_{U_{r}}^{p}(Y, \mathbb{R}) / \mathfrak{I}_{0}^{(p)}\left(U_{r}\right)$, which we shall briefly denote by $\mathscr{F}_{r}^{p}(Y, \mathbb{R}) / \mathscr{I}_{0}^{(p)}\left(Y_{r}\right)$. 
(We denote the corresponding sheaves by the calligraphic characters). There can always be found, at least locally, a representative $\lambda$, semi-basic with respect to the projection $\pi^{r}: Y_{r} \rightarrow Z$, that is $\lambda \in \mathscr{F}_{r}^{p}(Z, \mathbb{R})$. The Euler-Lagrange expressions which correspond to the Lagrange density $v_{J}{ }^{-1 \star} \lambda \in \mathscr{F}_{r}{ }^{p}\left(\mathbb{R}^{p}, \mathbb{R}\right)$ naturally take the shape of the components of the local expression of some differential $p$-form

$$
\varepsilon \in \mathscr{F}_{2 r}^{p}\left(Z, V_{0}^{*}\right)
$$

corresponding to the lagrangian $\lambda[4,5]$.

The symmetries of the Euler-Lagrange equations are nothing else but the symmetries of the system $\left(\mathfrak{S}_{\varepsilon}, \boldsymbol{\vartheta}_{2 r-1}\right)$, where the module $\mathfrak{S}_{\varepsilon}$ is generated by the vector bundle valued differential form $\boldsymbol{\varepsilon}$. From here on we shall deal with variational calculus in one independent variable. As an example we consider a system of third-order ordinary differential equations

$$
\mathrm{E}_{a}=0
$$

Let $Y=\mathbb{R}^{p} \times \mathbb{R}^{q}$, and let the canonical coordinates in the manifold $J_{r}\left(\mathbb{R}^{1} ; \mathbb{R}^{q}\right)$ be denoted by $t, \mathbf{x}=\left(\mathrm{x}^{a}\right), \mathbf{v}=\left(\mathbf{v}^{a}\right), \mathbf{v}^{\prime}=\left(\mathbf{v}^{a}\right), \ldots, \mathbf{v}^{(r-1)}=\left(\mathbf{v}^{(r-1)}\right)$.

This corresponds to $p=1, k=q$ in (2). We introduce a vector valued differental one-form

$$
\boldsymbol{\epsilon}=\mathrm{E}_{a} \mathbf{d} \mathbf{x}^{a} \otimes \mathbf{d} t,
$$

where the expressions $\mathrm{E}_{a}$ are the Euler-Poisson expressions. Applying the general criterion of [6] for an arbitrary system of differential equations to be a system of Euler-Poisson equations, it was established in $[7,8]$ that the vector expression $\mathbf{E}=$ $\left\{\mathrm{E}_{a}\right\}$ in (3) must take the shape of

$$
\mathbf{E}=\mathbb{A} \cdot \mathbf{v}^{\prime \prime}+\left(\mathbf{v}^{\prime} \cdot \partial_{\mathrm{v}}\right) \mathbb{A} \cdot \mathbf{v}^{\prime}+\mathbb{B} \cdot \mathbf{v}^{\prime}+\mathbf{c}
$$

where the skew-symmetric matrix $\mathbb{A}$, the matrix $\mathbb{B}$, and the column vector $\mathbf{c}$ depend on the variables $t, \mathbf{x}, \mathbf{v}=d \mathbf{x} / d t$, and satisfy the following system of partial differential equations in $t, \mathbf{x}^{a}$, and $\mathbf{v}^{a}[7,9]$

$$
\begin{aligned}
& \partial_{\mathrm{v}}\left[a \mathrm{~A}_{b c]}=0\right. \\
& 2 \mathrm{~B}_{[a b]}-3 \mathbf{D}_{1} \mathrm{~A}_{a b}=0 \\
& 2 \partial_{\mathbf{v}[a} \mathrm{B}_{b] c}-4 \partial_{\mathrm{x}^{[a}} \mathrm{A}_{b] c}+\partial_{\mathrm{x}^{c}} \mathrm{~A}_{a b}+2 \mathrm{D}_{\mathbf{1}} \partial_{\mathrm{v}^{c}} \mathrm{~A}_{a b}=0 \\
& \partial_{\mathbf{v}}\left({ }_{a} \mathbf{c}_{b)}-\mathbf{D}_{1} \mathbf{B}_{(a b)}=0\right. \\
& 2 \partial_{\mathbf{v}^{c}} \partial_{\mathbf{v}^{[a}} \mathrm{c}_{b]}-4 \partial_{\mathbf{x}^{[a}} \mathrm{B}_{b] c}+\mathbf{D}_{1}{ }^{2} \partial_{\mathbf{v}^{c}} \mathrm{~A}_{a b}+6 \mathbf{D}_{1} \partial_{\mathbf{x}^{[a}} \mathrm{A}_{b c]}=0 \\
& 4 \partial_{\mathbf{x}^{[a}} \boldsymbol{c}_{b]}-2 \mathbf{D}_{1} \partial_{\mathbf{v}}\left[a c_{b}\right]-\mathbf{D}_{1}^{3} \mathbf{A}_{a b}=0 \text {. }
\end{aligned}
$$

In (6) $\mathbf{D}_{\mathbf{s}}$ denotes the generators of Cartan distribution,

$$
\begin{aligned}
\mathrm{D}_{2}=\mathbf{v}^{\prime} . \partial_{\mathrm{v}}+ & \mathrm{D}_{1}, \\
& \mathbf{D}_{1}=\partial_{t}+\mathbf{v} . \boldsymbol{\partial}_{\mathrm{x}} .
\end{aligned}
$$


Let $\boldsymbol{\vartheta}_{2}, \boldsymbol{\vartheta}_{3}$ denote the canonical contact forms

$$
\begin{aligned}
\boldsymbol{\vartheta}_{3}=\frac{\partial}{\partial \mathbf{v}^{\prime a}} \otimes\left(\mathbf{d v}^{\prime a}-\mathrm{v}^{\prime \prime} \mathbf{d} t\right)+ & \boldsymbol{\vartheta}_{2}, \\
& \boldsymbol{\vartheta}_{\mathbf{2}}=\frac{\partial}{\partial \mathbf{v}^{a}} \otimes\left(\mathbf{d} \mathbf{v}^{a}-\mathbf{v}^{\prime a} \mathbf{d} t\right)+\frac{\partial}{\partial \mathbf{x}^{a}} \otimes\left(\mathbf{d} \mathrm{x}^{a}-\mathbf{v}^{a} \mathbf{d} t\right) .
\end{aligned}
$$

Along with the differential form $\boldsymbol{\epsilon}$, we introduce another one,

$$
\begin{array}{r}
\underline{\boldsymbol{\epsilon}}=\mathrm{A}_{a b} \mathbf{d} \mathrm{x}^{a} \otimes \mathbf{d v}^{\prime b}+\mathrm{K}_{a} \mathbf{d} \mathbf{x}^{a} \otimes \mathbf{d} t, \\
\quad \mathbf{K}=\left(\mathbf{v}^{\prime} . \partial_{\mathbf{v}}\right) \mathbb{A} \cdot \mathbf{v}^{\prime}+\mathbb{B} \cdot \mathbf{v}^{\prime}+\mathbf{c} .
\end{array}
$$

Exterior differential systems, generated by the forms $\boldsymbol{\epsilon}$ and $\underline{\boldsymbol{\epsilon}}$, are equivalent:

$$
\underline{\boldsymbol{\epsilon}}-\boldsymbol{\epsilon}=\boldsymbol{\vartheta}_{3} \bar{\wedge}\left(\mathrm{A}_{a b} \mathbf{d} \mathrm{x}^{a} \otimes \mathbf{d v}^{\mathbf{\prime}^{b}}\right) .
$$

Now it is time to put in the concept of symmetry. Let

$$
\mathfrak{x}=\mathfrak{t} \frac{\partial}{\partial t}+\mathfrak{x}^{a} \frac{\partial}{\partial \mathbf{x}^{a}}
$$

denote a generator of some local group of transformations of the manifold $\mathbb{R} \times \mathbb{R}^{q}$, its successive prolongations to the space $J_{s}\left(\mathbb{R} ; \mathbb{R}^{q}\right)$ denoted by $\mathfrak{x}_{s}$,

$$
\mathfrak{x}_{2}=\mathfrak{v}^{a} \frac{\partial}{\partial \mathfrak{v}^{\prime a}}+\mathfrak{x}_{1} .
$$

The demand that the exterior differential system, generated by the vector valued differential form $\underline{\boldsymbol{\epsilon}}$, be invariant under the infinitesimal transformation $\mathfrak{x}$ incarnates in consistency with (1) into the following equation

$$
\mathbf{L}\left(\mathfrak{x}_{2}\right)(\underline{\boldsymbol{\epsilon}})=\boldsymbol{\Xi} . \underline{\boldsymbol{\epsilon}}+\boldsymbol{\vartheta}_{2} \bar{\wedge} \boldsymbol{\omega},
$$

where the elements of a matrix $\boldsymbol{\Xi} \in \mathfrak{F}^{0}\left(J_{2}\left(\mathbb{R} ; \mathbb{R}^{q}\right) ; \operatorname{Hom}\left(\mathbb{R}^{q *} ; \mathbb{R}^{q *}\right)\right)$ and the coefficients of a $\pi_{1}^{2}$-horizontal $\mathbb{R}^{q *}$-valued one-form $\boldsymbol{\omega} \in \mathfrak{F}_{2}^{1}\left(J_{1}\left(\mathbb{R} ; \mathbb{R}^{q}\right) ; \mathbb{R}^{q *}\right)$ depend upon the variables $t, \mathbf{x}, \mathbf{v}$, and $\mathbf{v}^{\prime}$. Both $\boldsymbol{\Xi}$ and $\boldsymbol{\omega}$ play the role of Lagrange multipliers. Splitting equation (9) with respect to independent differentials $\mathbf{d} t, \mathbf{d} \mathbf{x}^{a}, \mathbf{d v}^{a}$, and $\mathbf{d v}^{\prime a}$, gives the following system of partial differential equations

$$
\begin{aligned}
\mathbf{L}\left(\mathfrak{x}_{1}\right) \mathrm{A}_{a b} & =\Xi_{a}^{c} \mathrm{~A}_{c b}-\mathrm{A}_{a c} \frac{\partial}{\partial \mathfrak{v}^{\prime b}} \mathfrak{v}^{c} \\
\mathbf{L}\left(\mathfrak{x}_{2}\right) \mathbf{K}_{a} & =\Xi_{a}{ }^{b} \mathrm{~K}_{b}-\mathrm{A}_{a b} \mathbf{D}_{2} \mathfrak{v}^{b}-\mathrm{K}_{a} \mathbf{D}_{\mathbf{1}} \mathfrak{t} .
\end{aligned}
$$


Variational problems in parametric form. A variational problem in parametric form is a variational problem, posed on the manifold $J_{r}(Z, M)$, so we have to put $Y=Z \times M$, the dimension of the manifold $M$ equal to $p+q$ with $p=1$, and $k=p+q=1+q$ in (2). Canonical coordinates in the manifold $J_{r}\left(\mathbb{R} ; \mathbb{R}^{1+q}\right)$ are denoted by $\tau, \boldsymbol{x}=\left(x^{\rho}\right), \boldsymbol{u}=\left(u^{\rho}\right), \dot{\boldsymbol{u}}=\left(\dot{u}^{\rho}\right), \ldots, \stackrel{r}{\boldsymbol{u}}^{-1}=\left({ }^{r-1}{ }^{\rho}\right)$. The manifold of $r^{\text {th }}$-order velocities, $T_{r}^{1} M$, is defined as $T_{r}^{1} M=J_{r}(\mathbb{R} ; M)_{0}$. There exists an obvious isomorphism $J_{r}(\mathbb{R} ; M) \approx \mathbb{R} \times T_{r}^{1} M$. Coordinates in the manifold $T_{r}^{1} M$ are denoted by $\boldsymbol{x}, \boldsymbol{u}, \dot{\boldsymbol{u}}, \ldots, \stackrel{r-1}{\boldsymbol{u}}$. If $M$ is a (pseudo-)Euclidean $n$-dimensional space (of an arbitrary signature), the Hodge operator " $*$ " is defined as $(* w)_{\rho_{k+1} \ldots \rho_{n}}=\frac{1}{(n-k) !} e_{\rho_{1} \ldots \rho_{n}} w^{\rho_{1} \ldots \rho_{k}}$.

Consider now a variational problem in parametric form, set by a Lagrangian

$$
\ell\left(\tau, x^{\rho}, u^{\rho}, \ldots,{ }^{r-1} \rho\right) \mathbf{d} \tau
$$

on the space $J_{r}(\mathbb{R} ; M)$. As long as we constrain ourselves only to the case of autonomous Euler-Poisson equations,

$$
\mathcal{E}_{\rho}=0
$$

the differential form

$$
\varepsilon=\mathcal{E}_{\rho} \mathbf{d} x^{\rho} \otimes \mathbf{d} \tau
$$

may globally be deprived of the factor $\mathbf{d} \tau$, constituting thus a globally defined $T^{*} M$ valued density

$$
e=\mathcal{E}_{\rho} \mathbf{d} x^{\rho} .
$$

Let $C_{r}^{1} M$ denote the manifold of the $r^{\text {th }}$ order contact germs of one-dimensional submanifolds of $M$. The projection $\wp: T_{r}^{1} M \backslash 0 \rightarrow C_{r}^{1} M$ can be employed to generate an autonomous variational problem set over $T_{r}^{1} M$ from every one variational problem over $C_{r}^{1} M$.

Lemma 2 ( [2]) In terms of a local chart, if in (4) the local semi-basic differential form $\boldsymbol{\epsilon}$ corresponds to the Lagrangian

$$
\lambda=L \mathbf{d} t,
$$

then the vector valued density

$$
\boldsymbol{e}=-u^{a}\left(\mathrm{E}_{a} \circ \wp\right) \mathbf{d} x^{0}+u^{0}\left(\mathrm{E}_{a} \circ \wp\right) \mathbf{d} x^{a}
$$

corresponds to the Lagrangian

$$
\ell\left(\tau, x^{\rho}, u^{\rho}, \ldots,,^{r-1} \rho\right) \mathbf{d} \tau=\mathcal{L}\left(x^{\rho}, u^{\rho}, \ldots,{ }^{r-1} u^{\rho}\right) \mathbf{d} \tau
$$

with the Lagrange function

$$
\mathcal{L}=u^{0} L \circ \wp .
$$


4. The classical spinning particle. Let in (8) generator $\mathfrak{x}$ correspond to the (pseudo-)orthogonal transformations of a four-dimensional (pseudo-)Euclidean space. In this case, equations (10) have no solutions of the third order, that is to say, no invariant system of Euler-Poisson equations (3) consists of at least one equation of the third order.

Nevertheless, if we allow a vector parameter $s=\left(s^{0}, \mathbf{s}\right)$ (that is, a constant quantity, transforming as a four-vector under the action of the (pseudo-) orthogonal group) enter into expressions (5), then equations ( $6 \& 10$ ) turn out to possess a family of solutions, which depend on $s$ and actually contain some third-order derivatives. In [7,9-11] we succeeded to obtain the following one (in an unessential assumption $g^{00}=+1$ )

$$
\begin{aligned}
\mathbf{E}= & \frac{\mathbf{v}^{\prime \prime} \times\left(\mathbf{s}-s_{0} \mathbf{v}\right)}{\left[(1+\mathbf{v} \cdot \mathbf{v})\left(s_{0}{ }^{2}+\mathbf{s} \cdot \mathbf{s}\right)-\left(s_{0}+\mathbf{s} \cdot \mathbf{v}\right)^{2}\right]^{3 / 2}} \\
& -3 \frac{\left(s_{0}{ }^{2}+\mathbf{s} \cdot \mathbf{s}\right) \mathbf{v}^{\prime} \cdot \mathbf{v}-\left(s_{0}+\mathbf{s} \cdot \mathbf{v}\right) \mathbf{s} \cdot \mathbf{v}^{\prime}}{\left[(1+\mathbf{v} \cdot \mathbf{v})\left(s_{0}{ }^{2}+\mathbf{s} \cdot \mathbf{s}\right)-\left(s_{0}+\mathbf{s} \cdot \mathbf{v}\right)^{2}\right]^{5 / 2}} \mathbf{v}^{\prime} \times\left(\mathbf{s}-s_{0} \mathbf{v}\right) \\
& +m \frac{(1+\mathbf{v} \cdot \mathbf{v}) \mathbf{v}^{\prime}-\left(\mathbf{v}^{\prime} \cdot \mathbf{v}\right) \mathbf{v}}{(1+\mathbf{v} \cdot \mathbf{v})^{3 / 2}\left(s_{0}{ }^{2}+\mathbf{s} \cdot \mathbf{s}\right)^{3 / 2}} .
\end{aligned}
$$

Applying the prescription (15) the above expression (17) produces the corresponding four-dimensional expression and thus the desired Euler-Poisson equations

$$
\mathcal{E}=\frac{* \ddot{\boldsymbol{u}} \wedge \boldsymbol{u} \wedge \boldsymbol{s}}{\|\boldsymbol{s} \wedge \boldsymbol{u}\|^{3}}-3 \frac{* \dot{\boldsymbol{u}} \wedge \boldsymbol{u} \wedge \boldsymbol{s}}{\|\boldsymbol{s} \wedge \boldsymbol{u}\|^{5}}(\dot{\boldsymbol{u}} \wedge \boldsymbol{s}) \cdot(\boldsymbol{u} \wedge \boldsymbol{s})+\frac{m}{\|s\|^{3}}\left[\frac{\dot{\boldsymbol{u}}}{\|\boldsymbol{u}\|}-\frac{\dot{\boldsymbol{u}} \cdot \boldsymbol{u}}{\|\boldsymbol{u}\|^{3}} \boldsymbol{u}\right]=\mathbf{0}
$$

Assume $\mathfrak{x}_{3}$ denote the third-order prolongation of the generator $\mathfrak{x}(8)$ of (pseudo-) orthogonal transformations with the group parameters $\mathbf{n}$, q to the space $J_{3}\left(\mathbb{R} ; \mathbb{R}^{3}\right)$,

$$
\begin{aligned}
\mathfrak{x}_{3} & =-(\mathbf{q} \cdot \mathbf{s}) \partial_{s^{0}}+s^{0}\left(\mathbf{q} \cdot \boldsymbol{\partial}_{\mathbf{s}}\right)+\left[\mathbf{n}, \mathbf{s}, \boldsymbol{\partial}_{\mathbf{s}}\right]-(\mathbf{q} \cdot \mathbf{x}) \partial_{t}+t\left(\mathbf{q} \cdot \boldsymbol{\partial}_{\mathbf{x}}\right)+\left[\mathbf{n}, \mathbf{x}, \boldsymbol{\partial}_{\mathbf{x}}\right] \\
& +\left(\mathbf{q} \cdot \boldsymbol{\partial}_{\mathbf{v}}\right)+(\mathbf{q} \cdot \mathbf{v})\left(\mathbf{v} \cdot \boldsymbol{\partial}_{\mathbf{v}}\right)+\left[\mathbf{n}, \mathbf{v}, \boldsymbol{\partial}_{\mathbf{v}}\right] \\
& +2(\mathbf{q} \cdot \mathbf{v})\left(\mathbf{v}^{\prime} \cdot \boldsymbol{\partial}_{\mathbf{v}^{\prime}}\right)+\left(\mathbf{q} \cdot \mathbf{v}^{\prime}\right)\left(\mathbf{v} \cdot \boldsymbol{\partial}_{\mathbf{v}^{\prime}}\right)+\left[\mathbf{n}, \mathbf{v}^{\prime}, \boldsymbol{\partial}_{\mathbf{v}^{\prime}}\right]
\end{aligned}
$$

The following assertions are true:

1. Vector quantity $\mathcal{E}$ in (18) constitutes a system of Euler-Poisson expressions;

2. Let vector field $\mathfrak{x}_{3}$ be given by (19), and let $\mathbf{E}$ be given by (17) (recall that (17) is nothing more but merely (18), parametrized with respect to the coordinate time $\left.t=x^{0}\right)$. The relation

$$
\mathbf{L}\left(\mathfrak{x}_{3}\right)(\mathbf{E})=\mathbf{n} \times \mathbf{E}+(\mathbf{q} \cdot \mathbf{v}) \mathbf{E}-(\mathbf{v} \cdot \mathbf{E}) \mathbf{q}
$$

proves the invariance of the Euler-Poisson equations (18), and at the same time shows that whatever a possible Lagrange function for (18) might exist, it by no means will reveal invariance under (pseudo-)orthogonal transformations, even in the generalized sense (that is up to a total derivative term). 
3. Equations (18) describe (in metric signature 2) the motion of relativistic spinning free particle with constant spin four-vector $\boldsymbol{s}$ and with the rest mass

$$
m_{0}=m\left[1-\frac{(\boldsymbol{s} \cdot \boldsymbol{u})^{2}}{(\boldsymbol{s} \cdot \boldsymbol{s})(\boldsymbol{u} \cdot \boldsymbol{u})}\right]^{3 / 2} .
$$

A comment on the order of the Euler-Lagrange form. Euler-Lagrange equations are polynomial with respect to the derivatives of orders greater than the maximal order of the derivatives which enter in the corresponding Lagrange function. It was this property that inspired us to try to diminish the order of the underlying manifold $J_{s}\left(\mathbb{R}, \mathbb{R}^{q}\right)$ from $s=3$ to $s=2$ by means of introducing the differential form $\underline{\epsilon}$ in (7) rather than $\boldsymbol{\epsilon}$.

\section{References}

[1] L.V. Ovsiannikov. Group Analysis of Differential Equations.- Orlando: Academic Press, 1982.- 416 pp.

[2] R.Ya. Matsyuk Exterior differential equations in generalized mechanics and symmetry properties. In bk.: Methods for Studying Differential and Integral Operators.- Kyiv: Naukova Dumka, 1989, p. 153-160. (In Russian). MR1109955(92b:58009).

[3] I. Kolář. Lie derivatives and higher-order Lagrangians. In bk.: Proceedings of the Conference (ČSŠR-GDR-Poland) on Differential Geometry and its Applications, September, 1980.- Praha: Univ. Karlova, 1981, p. 117-123.

[4] R.Ya. Matsyuk. Geometrical meaning of the integration by parts. In bk.: Lobachevskij and the Contemporary Geometry. The International Scientific Conference. Kazan', August 18-22. Thesis. Part I.- Kazan': Kazan' State Univ., 1992, p. 60-61. (In Russian).

[5] R.Ya. Matsyuk. Integration by parts and vector differential forms in higher order variational calculus on fibred manifolds. Matematychni Studii (Matematichn̄i Studìi). Vol. 11, no. 1, 1999, p. 85-107. MR1686048(2000b:58031). arXiv:1406.3369.

[6] W.M. Tulczyjew. The Euler-Lagrange resolution. In bk.: Differential Geometrical Methods in Mathematical Physics. Proc. conf. 1979. [Lecture Notes in Mathematics, 836].- - Berlin e.a: Springer-Verlag, 1980, p. 22-48.

[7] R.Ya. Matsyuk. Poincaré-invariant Equations of Motion in Lagrangian Mechanics With Higher Derivatives. Ph.D. thesis, Institute for Applied Problems in Mechanics and Mathematics, Academy of Science. Ukraine.- L'viv, 1984.140 pp. (In Russian). 
[8] R.Ya. Matsyuk. Existence of a Lagrangian for a nonautonomous system of ordinary differential equations. Mat. Metody i Fiz.-Mekh. Polya. Issue 20.- Kyiv, 1984, p. 16-19. (In Russian). MR0756973(85g:70020), Zbl 0536.34007.

[9] R.Ya. Matsyuk. Lagrangian analysis of the third-order invariant equations of motion in the relativistic mechanics of classical particles. Sov. Phys.-Dokl. (U.S.A.). Vol. 30, no. 6, 1985, p. 458-460. MR0802859(87d:70028).

[10] R.Ya. Matsyuk. Lagrangian approach to spinning or radiating particle higherorder equations of motion in special relativity. In bk.: $11^{\text {th }}$ International Conference on General Relativity and Gravitation. Stockholm, Sweeden, July 612, 1986. Abstracts of contributed papers, Vol. II.- Stockholm, 1986, p. 648.

[11] Roman Matsyuk. Third order variational equation for the free relativistic top. Fizyčnyj zbirnyk NTŠ. Collected physical papers of the NTSh. Vol. 9.Shevchenko Scientific Society: L'viv, 2014. (in Ukrainian, to appear). 